\title{
Sensorineural hearing loss as an exceptional mode of revelation of nasopharyngeal carcinoma: A case report
}

\author{
Hind Temsamani, ", Ahmed Belkouch ${ }^{2}$, Abdelilah Mouhsine ${ }^{3}$, Ali Jahidi ${ }^{1}$, Abdelghani El Fikri ${ }^{3}$, \\ Lahcen Belyamani ${ }^{2}$, Fouad Benariba ${ }^{1}$ \\ ${ }^{1}$ Department of ENT and Cervico Facial Surgery. MohamedV Military Hospital of Instruction, Faculty of Medicine and Pharmacy, Rabat, \\ Morocco \\ ${ }^{2}$ Emergency Department, MohamedV Military Hospital of Instruction, Faculty of Medicine and Pharmacy, Rabat, Morocco \\ ${ }^{3}$ Department of Radiology, Avicenna Military Hospital, Faculty of Medicine and Pharmacy, Marrakech, Morocco
}

\section{Email address:}

htemsamani@gmail.com (H. Temsamani), belkouch1@gmail.com (A. Belkouch)

\section{To cite this article:}

Hind Temsamani, Ahmed Belkouch, Abdelilah Mouhsine, Ali Jahidi, Abdelghani El Fikri, Lahcen Belyamani, Fouad Benariba. Sensorineural Hearing Loss as an Exceptional Mode of Revelation of Nasopharyngeal Carcinoma: A Case Report. Cancer Research Journal.

Vol. 2, No. 6, 2014, pp. 104-107. doi: 10.11648/j.crj.20140206.12

\begin{abstract}
Nasopharyngeal carcinomas are responsible of a high mortality rate in many parts of the world. The clinical aspects of these cancers are various; they find their explanations in the ways of tumor extension. The knowledge of these expansion ways has been significantly modified by the addition of CT and MRI. To the best of our knowledge, this is the first case of nasopharyngeal carcinomas that manifested with sensorineural hearing loss as the first and unique symptom. We report the case of a 48 years old male Arab patient with an unusual mode of revelation of this cancer, he presented with sensorineural hearing loss. He had no other clinical manifestations. The patient was assessed for Cerebellopontine Angle Tumors, MRI rectified the diagnosis. The intracranial extension of nasopharyngeal carcinomas may be manifested by sensorineural hearing loss due to the auditory nerve damage and can be the first and the unique symptom of the disease. Imaging section (CT scan and MRI) is the cornerstone that can rectify the diagnosis.
\end{abstract}

Keywords: Nasopharyngeal Carcinoma, Sensorineural Hearing Loss, Tumor Extension

\section{Introduction}

Morocco is one of the endemic countries of nasopharyngeal carcinoma with an intermediate incidence of 4 to 8 new cases per 100,000 people per year $(1,2)$. The first clinical signs are usually late-onset and associated with the invasion of the neighboring structures. The contribution of imaging in recent decades has revolutionized the diagnosis. It also facilitated the study of tumor extension and explaining the different clinical manifestations. We report a unique case of nasopharyngeal carcinoma revealed by a sensorineural hearing loss.

\section{Case Presentation}

A 48years old male patient, known diabetic since eight years treated by oral antidiabetics, presented with an insidious left hearing loss that began two months ago. The patient did not report tinnitus, or fullness in the ear, nor autophony or sensations of liquid changing place, and he did not complain of vertigo or instability, or headache. There was no sensation of nasal obstruction or epistaxis, or rhinorrhea.

On examination the left eardrum was slightly frosted without visible fluid level, it was mobile in Valsalva maneuver Palpation did not found lymphadenopathy in the cervical and spinal regions. The cranial nerves were intact and neurological examination showed no abnormalities. The Rinne was positive and the Weber lateralized to the right. The tone audiometry objectified a left sensorineural hearing loss between 90 and $110 \mathrm{~dB}$ on all frequencies. Tympanometry showed a plot of curve flattened with a prominent peak type AS, and the study of acoustic reflex showed fatigability. Nasofibroscopy found a slight asymmetry of the nasopharynx without altering the lining which initially was not considered.

Given these results and due to delayed auditory evoked potential study, MRI was requested and objectified tissular lesion centered on the Rosen Muller fossa stretching back to 
the pre-vertebral muscles, infiltrating the body of the sphenoid and clearing the para pharyngeal left space. Above, it extends to the endocranium through the peri carotid foramen lacerum with nerve extension to the hole of the hypoglossal nerve and mixed nerves into the posterior fossa. This lesion enhances intensely with gadolinium and measures $40 \times 37 \times 30 \mathrm{~mm}$ (figures 1a, b, c).

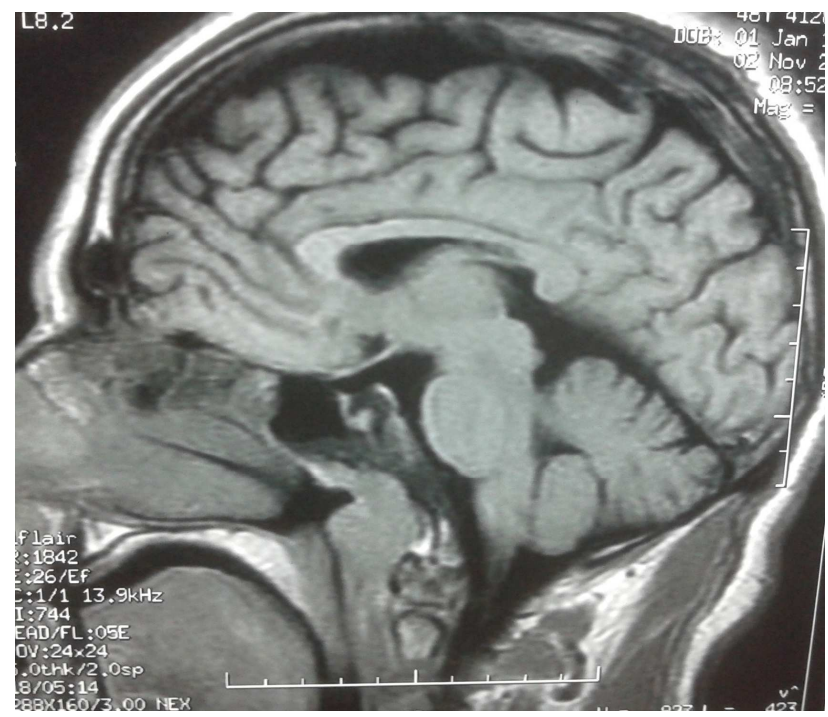

Figure 1a. MRI T1 weighted flair along a sagittal plan showing a lesion of posterior superior wall of the nasopharynx.

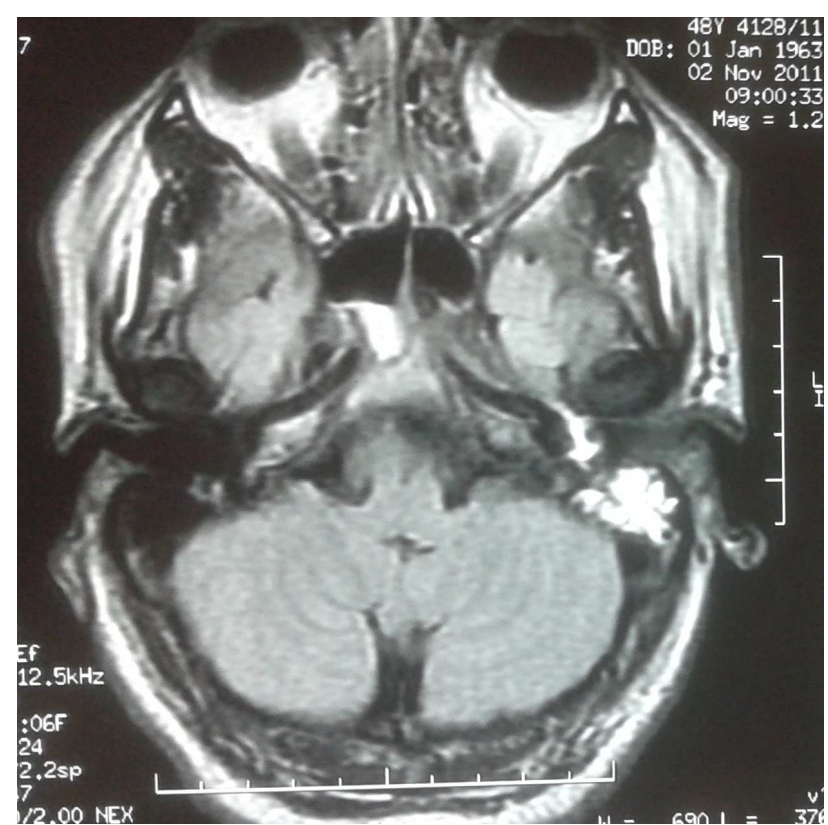

Figure 1b. MRI T2-weighted sequence flair in an axial plan showing a large heterogeneous tissular lesion of the posterior superior and lateral wall of the left center of the Rosen Müller fossa and Eustachian tube which are invaded. Associated to This process, in the ipsilateral ear, it shows a mastoid inflammatory filling. This process extends back to the pre-vertebral muscles and penetrates the body of the sphenoid, and it clears the left para pharyngeal space, then, it extends upward through the foramen lacerum.

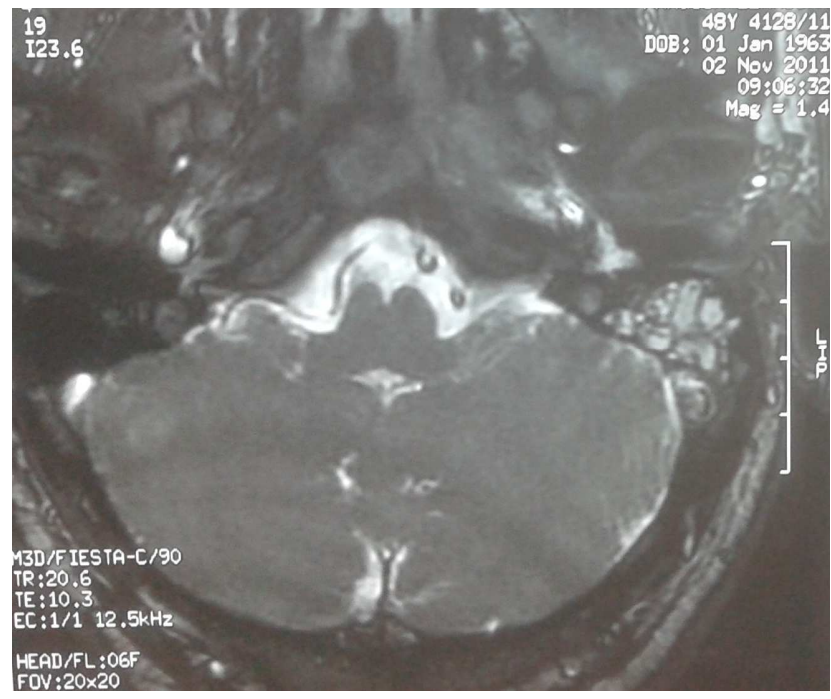

Figure 1c. fiesta axial sequence showing the above described process invading the left acoustic-facial package with ipsilateral mastoid ear filling.

Choanae, oro and hypopharinx were free. We also noted the presence of necrotic retro pharyngeal lymphadenopathy and spinal measuring $33 \mathrm{~mm}$ at long axis, concluding to a nasopharyngeal lesion classified T4N2 with mastoid filling process.

Nasopharyngeal and cervical-thoracic-abdominal-pelvic CTscan confirmed the diagnosis (figure 2), classifying the tumor T4N2M0. We performed an endoscopy with biopsy deep in the Cavum which showed, a cancerous process poorly differentiated, in the pathological study, with strong immunohistochemistry expression of CKAE1 / AE23.

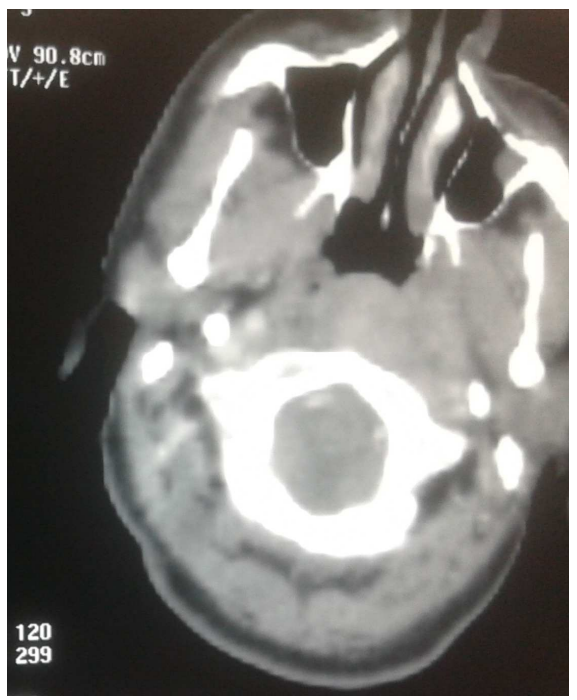

Figure 2. CT axial cut objectifying tissular density process of the upper posterior wall of the nasopharynx lateralized to the left side effacing the Rosen Müller fossa and Eustachian tube.

The outcome was favorable after chemoradiotherapy and the patient is declared in remission to date. Regarding his hearing, recovery was partial and now he uses a hearing aid to facilitate his daily life. 


\section{Discussion}

Nasopharyngeal cancer continues to draw attention, due to its incidence. It is endemic in parts of Southeast Asia and it affects 30-80 / 100,000 people per year (1). The countries of North Africa and Arabia are also experiencing a high incidence 4/100 000 (2).

In addition, because of the nonspecific nature of the symptoms and the difficulty of accessing the nasopharynx to the clinical examination, most patients with this disease are diagnosed when the tumor has reached an advanced stage (stages III and IV) (3).

Indeed, nasopharyngeal tumors can occur in four clinical presentations:

- Naso pharyngeal symptoms: epistaxis, nasal obstruction and purulent discharge.

- Tubal dysfunction: deafness and tinnitus due to progressive transmission hearing loss (4).

- Cranial nerves disorders: headache, diplopia, facial pain and tingling sensations ...

- Neck mass (3).

In a retrospective analysis of 4768 patients with nasopharyngeal cancer, the clinical signs in the onset were as follows: cervical mass $(76 \%)$, nasal pharyngeal symptoms (73\%), hearing loss $(62 \%)$, headache $(35 \%)$, diplopia $(11 \%)$, facial numbness $(8 \%)$, weight loss $(7 \%)$ and lockjaw $(3 \%)$. The physical signs at diagnosis were enlarged neck node $(75 \%)$ and cranial nerve palsy $(20 \%)$. The cranial nerves most commonly affected are the third, fifth, sixth and 12 th nerves $(5$, 6 ). The symptoms in young patients were generally similar to those reported in adults (7).

Tumor extension clearly explains the pathogenesis of each form of disease onset. It has been improved through the contribution of the brain and cervical CT scan with injection of contrast material (8). MRI is more efficient to detail the marrow involvement, muscular, nervous and intracranial perished (9).

This tumor extension can follow three ways (10):

- In $20 \%$ of cases, it follows the posterior and inferior axis with extension to the retro pharyngeal and pre vertebral spaces, and goes downward along the cervical axis in theory until mediastinum. It can seep further back in the vidian canal and extend into the foramen lacerum and the sphenoid sinus.

- Direct upper axis is possible by bone erosion of the sphenoid bone.

- The most common remains posterolateral axis that is invaded in $80 \%$ of cases. The superior constrictor muscle of the pharynx, prolonged by its pharyngo basilar fascia, obstructs this way. However, it leaves passage to the levator veli palatini which is the elevator muscle of the soft palate and to the torus tobarius, cartilaginous portion of the Eustachian tube, which use the sinus Morgagni to extend. Permeability disorders of the tube can then induce its dysfunction: feeling full ear, ear effusion, effusion mastoid air cells resulting in hearing loss. Once that hurdle crossed, the tumor can invade therefore:
- The parapharyngeal space, and follows the craniocaudal plan.

- The carotid space, the tumor can then go upward through the jugular foramen or carotid canal and become intracranial. Intracranial involvement is rare $(<10 \%)$

Further, the tumor extends forward (and sideways) to reach the pterygoid muscles, and reach the pterygomaxillary fossa and back into the orbit along the inferior orbital fissure. It can invade the masticator space and move up to the foramen ovale more laterally and invade the trigeminal ganglion and the cavernous nerves and the nearby sinus (III, IV, VI).

Finally, the nodal disease is often bilateral ( $50 \%$ of cases). The areas most frequently invaded are II, III, IV and retro pharyngeal, but all can be involved, including the area $\mathrm{V}$.

In our case, the tumor developed in posterior and became intracranial. It manifested solely and exclusively by sensorineural deafness by compression or invasion of the acoustic nerve. To our knowledge this is the first case of nasopharyngeal carcinoma manifested by sensorineural hearing loss.

\section{Conclusion}

The ways of extending of nasopharyngeal carcinoma explain many Clinical symptoms. The intracranial extension may be manifested by sensorineural hearing loss due to damage to the auditory nerve and can be the first symptom of the disease. Imaging section (CT scan and MRI) is the cornerstone that can rectify the diagnosis.

\section{References}

[1] Muir CS, Waterhouse J, Mack T: Cancer Incidence in Five Continents, vol. 5. IARC Sci. Publ. No. 88. Lyon, IARC, 1987.

[2] Johansen LV, Mestre M, Overgaard J: Carcinoma of the nasopharynx; analysis of treatment results in 167 consecutively admitted patients. Head Neck 1992, 14: 200-207.

[3] Wei W.I, Sham J.S.T: Nasopharyngeal carcinoma. Lancet, 2005, 365 (9476): 2041-2054.

[4] S. Riveraa,*, C. Keryer b, P. Busson b, P. Maingon a : Les carcinomes du nasopharynx : de la biologie à la clinique. Cancer/Radiothérapie 9 (2005) 55-68.

[5] Lee AWM, Foo W, Law SCK, et al: Nasopharyngeal carcinoma: presenting symptoms and duration before diagnosis. HK Med $J$ 1997; 3: 355-361.

[6] Ozyar E, Atahan IL, Akyol FH, Gurkaynak M, Zorlu AF: Cranial nerve involvement in nasopharyngeal carcinoma: its prognostic role and response to radiotherapy. Radiat Med 1994; 12: $65-68$.

[7] Sham JS, Poon YF, Wei WI, Choy D: Nasopharyngeal carcinoma in young patients. Cancer 1990; 65: 2606-2610.

[8] Chong VFH, Fan YF: Skull base erosion in nasopharyngeal carcinoma: detection by CT and MRI. Clin Radiol 1996; 51: 625-31. 
[9] Eisen MD, Youssem DM, Montone T: Use of preoperative MRI to predict dural, perineural and venous sinus invasion of skull base tumors. Am J Neuroradiol 1996; 17: 1937-45.
[10] P.Y. Marcy, Y. Zhu, R.J. Bensadoun: Target volumes in radiotherapy - head and neck tumors intensity - modulated radiation therapy (IMRT) of nasopharyngeal carcinoma: practical aspects in the delineation of target volumes and organs at risk. Cancer/Radiother, 9 (2005): 240-250. 